\title{
Exponential Stability of Linear Systems with Multiple Time Delays
}

*Ordokhani Y.; Faculty of Mathematical sciences, Alzahra University Taghizadeh E.; Faculty of Mathematical Sciences, University of Mazandaran Behmardi D.; Faculty of Mathematical sciences, Alzahra University Matin Far M.; Faculty of Mathematical Sciences, University of Mazandaran Received: 18 Nov 2013 Revised: 10 Nov 2014

\begin{abstract}
In this paper, a class of linear systems with multiple time delays is studied. The problem of exponential stability of time-delay systems has been investigated by using Lyapunov functional method. We will convert the system of multiple time delays into a single time delay system and show that if the old system is stable then the new one is so. Then we investigate the stability of converted new system, by using matrix decomposition and linear matrix inequality (LMI) technique. Some numerical examples are given to illustrate the efficiency of our method.
\end{abstract}

Keywords: Exponential stability, Multiple time-delays system, Matrix decomposition, LyapunovKrasovskii functional, LMI

\section{Introduction}

Time delays are often encountered due to measurment and computational delays, transmission and transport lags. Frequently, it is a source of the generation of oscillation and a source of instability in many biological and engineering systems. Therefore, stability testing and stabilization of time-delay systems are problems of practical and theoretical interest. Over the past years, considerable efforts have been devoted to the analysis of the stability or stabilization of systems with time delays and many different methods have been proposed to deal with the stability or stabilization problem, see for example, $[1,10]$.

There are many different methods dealing with the exponential stability problem. Among the well-known Lyapunov stability methods, the Lyapunov functional method is a powerful tool for studying system stability, even for linear systems. Numerous works

\footnotetext{
*Corresponding author: ordokhani@alzahra.ac.ir
} 
deal with the stability of linear systems using the Lyapunov function method [1]. Timedelay stability conditions for time-invariant systems were formulated in both algebraic Riccati equation and (LMI) $[1,3,4,5]$. Some stability conditions are directly obtained from the eigenvalues or the robust stability conditions in terms of matrix measures and norm criteria [6], or in terms of the solution of some algebraic Riccati equations [7].

We provide an overview of two application problems, inverted pendulum systems and angiogenesis models with discrete delays.

Example 1:( Inverted pendulum) Consider the inverted pendulum on a cart such as in [14]. The physical structure is shown in Figure. 1 (which is taken from [14]). In this system, a pendulum is attached to the side of a cart by means of a pivot which allows the pendulum to showing in the $x y$-plane. A force $F(t)$ is applied to the cart in the $x$ direction, with the purpose of keeping the pendulum balanced upright. We assume that the pendulum is modeled as a thin rod. Then, applying Newton's second law to the linear and angular displacement, we arrive at the equations of motion for the system

$$
\begin{aligned}
& (M+m) \ddot{x}+\varepsilon \dot{x}+m \ddot{\theta} l \cos \theta-m l \dot{\theta}^{2} \sin \theta=F(t), \\
& m l \ddot{x} \cos \theta+\frac{4}{3} m l^{2} \ddot{\theta}-m g l \sin \theta=0 .
\end{aligned}
$$

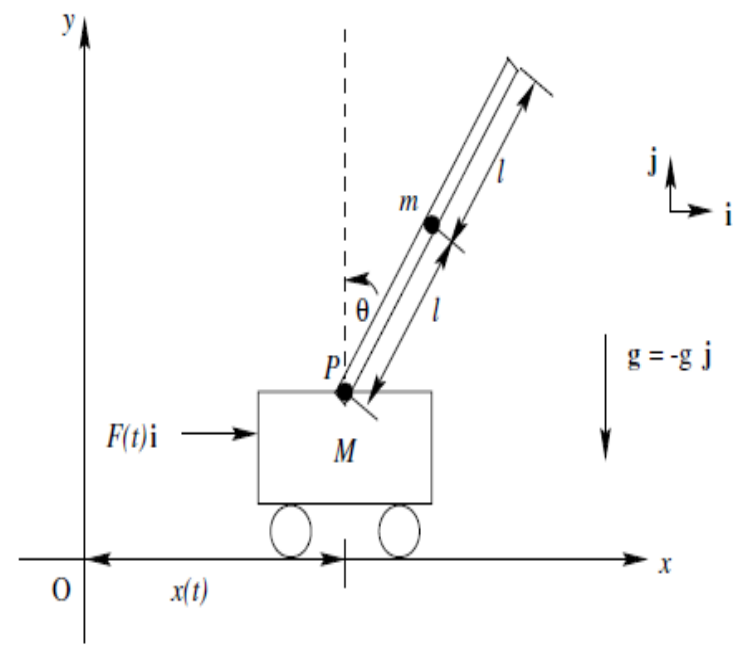

Figure1. A typical inverted pendulum fixed on a moving cart

A more convenient form of the equations is found by solving for $\ddot{x}$ and $\ddot{\theta}$ from equations (1) and (2). Introducing the variables

$$
y=\left(y_{1}, y_{2}, y_{3}, y_{4}\right)^{T}=(x, \theta, \dot{x}, \dot{\theta})^{T},
$$

with the delay $h$, it follows from [13] that the system can be rewritten in the form of

$$
\dot{y}(t)=A y(t)+B y(t-h),
$$


Table1. Notation of Example 1

\begin{tabular}{|l|l|}
\hline$x(t)$ & Displacement of the center of mass of the cart from point O \\
\hline$\theta(t)$ & Angle the pendulum makes with the top vertical \\
\hline$M$ & Mass of the cart \\
\hline$m$ & Mass of the pendulum \\
\hline$L$ & Length of the pendulum \\
\hline$l$ & Distance from pivot to the center of mass of the pendulum $l=\frac{L}{2}$ \\
\hline $\mathrm{P}$ & Pivot point of the pendulum \\
\hline$F(t)$ & Force applied to the cart \\
\hline
\end{tabular}

where

$$
\begin{gathered}
A=\left(\begin{array}{cccc}
0 & 0 & 1 & 0 \\
0 & 0 & 0 & 1 \\
0 & c_{1} & c_{2} & 0 \\
0 & c_{3} & c_{4} & 0
\end{array}\right), B=\left(\begin{array}{cccc}
0 & 0 & 0 & 0 \\
0 & 0 & 0 & 0 \\
e_{1} & e_{2} & e_{3} & e_{4} \\
e_{5} & e_{6} & e_{7} & e_{8}
\end{array}\right), \\
c_{1}=\frac{-3 m g}{(m+4 M)}, c_{2}=\frac{-3(\beta+\varepsilon)}{(m+4 M)}, c_{3}=\frac{3(m+M) g}{l(m+4 M)}, c_{4}=\frac{3(\beta+\varepsilon)}{l(m+4 M)}, \\
e_{i}=\frac{4 d_{i}}{(m+4 M)}, i=1, . ., 4, \quad e_{i}=\frac{-3 d_{i}}{l(m+4 M)}, i=5, . ., 8,
\end{gathered}
$$

And $\beta, \varepsilon$ are constant numbers.

Example 2: (Angiogenesis models with discrete delays) consider a family of models with delays describing the process of angiogenesis that is a physiological process involving the growth of new blood vessels from pre-existing ones. This family includes the well- known models of tumor angiogenesis proposed by Hahnfeldt et al. and Ergun et al. and is based on the Gompertz type of the tumor growth [15].

We consider the following system of differential equations with two discrete time delays describing the process of tumor angiogenesis

$$
\begin{gathered}
\frac{d}{d t} p(t)=-r p(t) \ln \left(\frac{p\left(t-h_{1}\right)}{q\left(t-h_{1}\right)}\right), \\
\frac{d}{d t} q(t)=q(t)\left[b\left(\frac{p\left(t-h_{2}\right)}{q\left(t-h_{2}\right)}\right)^{\alpha}-a p^{\frac{2}{3}}(t)-\mu\right],
\end{gathered}
$$


where $\mathrm{p}(\mathrm{t})$ and $\mathrm{q}(\mathrm{t})$ denote tumor volume and the density of endothelial cells at time t, respectively. We follow the Hahnfeldt et al. assumption in [15] that the tumor volume dynamics, described by Eq. (5), is governed by the Gompertz-type equation for which the carrying capacity of tumor cells is proportional to the density of endothelial cells. Eq (6) describes the dynamics of endothelial cells which depends on the stimulation process initiated by poorly nourished tumor cells, vessels lost due to the accumulation of the inhibiting factors secreted by tumor cells within the tumor [15]. Then from [15], the system can be rewritten in the form of

$$
\begin{gathered}
\left.\frac{d}{d t} x(t)=-r x\left(t-h_{1}\right)-y\left(t-h_{1}\right)\right), \\
\frac{d}{d t} y(t)=b e^{\alpha\left(x\left(t-h_{2}\right)-y\left(t-h_{2}\right)\right)}-(b-\mu) e^{\frac{2}{3} x(t)}-\mu .
\end{gathered}
$$

To close the system (7)-(8) we define an initial condition, that is a continuous function $\phi:[-h, 0] \rightarrow \mathbb{R}^{2}$ where $h=\max \left\{h_{i}: i=1,2\right\}$.

The linearization of Eq (7)-(8) it follows from [15] that the system can be rewritten in form of

$$
\left.\dot{z}(t)=A_{0} z(t)+\sum_{i=1}^{2} A_{i} z\left(t-h_{i}\right)\right)
$$

where

$$
z(t)=(x(t), y(t)) .
$$

In this paper we will convert a system of multiple time delays into a single time delay system and show that if the old system is stable, then the new one is so. Then we will consider the exponential stability problem for linear systems with single delay, based on the Lyapunov-Kradovskii functional approach and (LMI). Numerical examples are given to illustrate the efficiency of our method.

\section{Preliminaries}

In this section we state definitions and the required mathematical background, as well as the class of systems considered throughout the paper. First we introduce some elementary definitions and lemmas which play an important role in the proof of the main results.

We consider the following linear systems with multiple time delays, described by 


$$
\begin{gathered}
\dot{x}(t)=A_{0} x(t)+\sum_{i=1}^{m} A_{i} x\left(t-h_{i}\right), \quad t \in \mathbb{R}^{+}, \\
x(t)=\phi(t), \quad t \in[-h, 0],
\end{gathered}
$$

where $\quad h=\max \left\{h_{i}: i=1,2, \ldots, m\right\}, A_{i} \in M^{n \times r}, i=0,1,2, \ldots, m, \quad$ are $\quad$ constant $\quad$ and $\phi(t) \in C\left([-h, 0], \mathbb{R}^{n}\right), C\left([-h, 0], \mathbb{R}^{n}\right)$ denotes the Banach space of all piecewisecontinuous vector valued functions mapping $[-h, 0]$ into $\mathbb{R}^{n}$.

Definition 1 [8] The system (10) is said to be exponentially stable with decay rate $\alpha$, if there is a function $\eta: \mathbb{R}^{n} \rightarrow \mathbb{R}^{n}$ such that for each $\phi(t) \in C\left([-h, 0], \mathbb{R}^{n}\right)$, the solution $x($ $t, \phi)$ of the system satisfies $\|x(t, \phi)\| \leq \eta(\|A\|) e^{-\alpha t}$, for all $t \in \mathbb{R}^{+}$.

Define the operator $D\left(x_{t}\right): C\left([-h, 0], \mathbb{R}^{n}\right) \rightarrow \mathbb{R}^{n}$ as

$$
D\left(x_{t}\right)=x(t)+G_{0} \int_{t-h}^{t} x(s) d s,
$$

where $x_{t}(s)=x(t+s)$, and $G_{0} \in \mathbb{R}^{n \times n}$ is a constant matrix. We have the following fact about $D\left(x_{t}\right)$.

Lemma 1 [11] The operator (11) is stable if there exist a scalar $0<\delta<1$ and positive symmetric $M$ such that

$$
\left(\begin{array}{cc}
-\delta M & h G_{0}^{T} M \\
h M^{T} G_{0} & -M
\end{array}\right)<0
$$

Lemma 2 [11] Assume that $S \in M^{n \times n}$ is a symmetric positive definite matrix. Then for every $Q \in M^{n \times n}$ and $x, y \in \mathbb{R}^{n}$

$$
2\langle Q y, x\rangle-\langle S y, y\rangle \leq\left\langle Q S^{-1} Q^{T} x, x\right\rangle .
$$

Lemma 3 [12] Let $P, Q, S \in M^{n \times n}$ be given matrices such that $S>0$ and $S=S^{T}$. Then

$$
\left(\begin{array}{cc}
P & Q \\
Q^{T} & -S
\end{array}\right)<0 \Leftrightarrow P+Q S^{-1} Q<0 .
$$

Lemma 4 [12] Suppose $U$ and $V$ are real symmetric matrices with $U>0$ and $V \geq 0$.

Then

$$
U>V \Leftrightarrow \lambda_{\max }\left(V U^{-1}\right)<1 \Leftrightarrow \lambda_{\max }\left(U^{-1 / 2} V U^{-1 / 2}\right)<1
$$

where $\lambda_{\max }$ is maximal eigenvalues of a symmetric matrix.

Lemma 5 [12] Suppose $U$ and $V$ are real symmetric matrices with $U>0$ and $V \geq 0$.

Then there is a positive number $\sigma$ such that $-U+\sigma V<0$.

Lemma 6 [12] Suppose $U$ and $V$ are real symmetric matrices with $U>0$ and $V \geq 0$ and $a$ is a positive number. Then 


$$
a U>V \Leftrightarrow \lambda_{\max }\left(V U^{-1}\right)<a \Leftrightarrow \lambda_{\max }\left(U^{-\frac{1}{2}} V U^{-\frac{1}{2}}\right)<a .
$$

Two problems are considered in the rest of this paper. First, it is shown that if the system (10) is stable then the system (13) is so. Second, stability of linear system with single delay will be presented.

Theorem 1 [16] System (10) is uniformly asymptotically stable independent of delay if

$$
\mu\left(A_{0}\right)+\sum_{i=1}^{m}\left\|A_{k}\right\|<0 .
$$

Considering the time delay system described by (10), the matrices $A_{i}, i=1,2, \ldots, n$, are converted to diagonal form and subtract $\varepsilon>0$ from each diagonal entry. we choose the matrix with maximum norm which is denoted by $G_{\varepsilon}$. The remaining matrix is denoted by $B_{\varepsilon}$. Therefore from system (10) we have

$$
\begin{gathered}
\dot{x}(t)=A_{0} x(t)+\left(G_{\varepsilon}+m B_{\varepsilon}\right) x(t-h), t \in \mathbb{R}^{+}, \\
x(t)=\phi(t), t \in[-h, 0],
\end{gathered}
$$

where the matrix functions $A_{0},\left(G_{\varepsilon}+m B_{\varepsilon}\right)$ are constant matrices and $B=\left(G_{\varepsilon}+m B_{\varepsilon}\right)$.

Remark 1: To show the stability of the system (13), consider the time delay system described by (10). Then the matrices $A_{i}, i=1,2, \ldots, n$, are converted to diagonal form and subtracting $\varepsilon>0$ from each diagonal entry, we assume that the system with multiple time delay is stable and satisfies (12). We convert the system with multiple time delays to a stable system with single time-delay (13). Then, we know

$$
\left\|G_{\varepsilon}+m B_{\varepsilon}\right\| \leq\left\|G_{\varepsilon}\right\|+m\left\|B_{\varepsilon}\right\|<\left\|G_{1 \varepsilon}\right\|+\left\|G_{2 \varepsilon}\right\|+\ldots\left\|G_{m \varepsilon}\right\|+m\left\|B_{\varepsilon}\right\|,
$$

and using Theorem 1 we have

$$
\mu\left(A_{0}\right)+\left\|G_{\varepsilon}+m B_{\varepsilon}\right\|<\mu\left(A_{0}\right)+\sum_{i=1}^{m}\left\|A_{i}\right\|=\mu\left(A_{0}\right)+\sum_{i=1}^{m}\left\|G_{i \varepsilon}+m B_{\varepsilon}\right\|<0 .
$$

Hence system (13) is uniformily asymptotically stable independent of delay and we get the result.

\section{Main results}

In this section, exponential stability with decay rate $\alpha$ criterion for system (13) is derived by using Lyapunov functional and LMI approach.

Theorem 2 For delay time $h>0$, system(13) is exponentially stable with decay rate $\alpha$, if there exists symmetric and positive-definite matrices $P>0, Q>0$ such that the following LMIs hold: 


$$
\begin{gathered}
\left(\begin{array}{cc}
-I & h e^{\alpha h} B^{T} \\
h e^{\alpha h} B & -I
\end{array}\right)<0, \\
\left(\begin{array}{cc}
\bar{A}^{T} P+P \bar{A}+h Q & h e^{\alpha h} \bar{A}^{T} P B \\
h e^{\alpha h} B^{T} P \bar{A} & -h Q
\end{array}\right)<0,
\end{gathered}
$$

where $\bar{A}=B_{0}+B e^{\alpha h}$.

Proof. Consider the time delay systems (13) and use the following transformation:

$$
y(t)=e^{\alpha t} x(t)
$$

where $\alpha>0$ is the stability degree. To transform (13) to

$$
\dot{y}(t)=B_{0} y(t)+e^{\alpha t} B y(t-h),
$$

where $B_{0}=A_{0}+\alpha I$, consider the following Lyapunov- Krasovskii functional candidate :

$$
V(y(t))=D^{T}(y(t)) P D(y(t))+\int_{-h}^{0} \int_{t+s}^{t} y^{T}(\rho) Q y(\rho) d \rho d s,
$$

where $D(y(t))=y(t)+\int_{t-h}^{t} e^{\alpha h} B y(s) d s$.

Therefore we have

$$
\begin{gathered}
\dot{D}(y(t))=\dot{y}(t)+e^{\alpha h} B y(t)-e^{\alpha h} B y(t-h) \\
=B_{0} y(t)+e^{\alpha h} B y(t-h)+e^{\alpha h} B y(t)-e^{\alpha h} B y(t-h)=\left(B_{0}+e^{\alpha h} B\right) y(t) .
\end{gathered}
$$

Taking the time derivative of the Lyapunov functional along the trajectory of system (16), we have

$$
\begin{gathered}
\dot{V}(y(t))=\dot{D}^{T} P D+D^{T} P \dot{D}+y^{T}(t) h Q y(t)-\int_{t-h}^{t} y(s)^{T} Q y(s) d s=(\bar{A} y(t))^{T} P(y(t)+ \\
\left.\int_{t-h}^{t} B e^{\alpha h} y(s) d s\right)+\left(y(t)+\int_{t-h}^{t} B e^{\alpha h} y(s) d s\right)^{T} P \bar{A} y(t)+y(t)^{T} h Q y(t) \\
-\int_{t-h}^{t} y(s)^{T} Q y(s) d s \\
=y(t)^{T}\left(\bar{A}^{T} P+P \bar{A}+h Q\right) y(t)+2 y(t)^{T} \bar{A}^{T} P \int_{t-h}^{t} B e^{\alpha h} y(s) d s-\int_{t-h}^{t} y(s)^{T} Q y(s) d s .
\end{gathered}
$$

By assuming $2 a b \leq a^{T} Q^{-1} a+b^{T} Q b$, we have

$$
2 y(t)^{T} \bar{A}^{T} P \int_{t-h}^{t} B e^{\alpha h} y(s) d s \leq y(t)^{T} h e^{2 \alpha h} \bar{A}^{T} P B Q^{-1} B^{T} P \bar{A} y(t)+\int_{t-h}^{t} y(s)^{T} Q y(s) d s .
$$

Hence, we obtain $\dot{V}(y(t)) \leq y(t)^{T} S_{1} y(t)$, where

$$
S_{1}=\bar{A}^{T} P+P \bar{A}+h e^{2 \alpha h} \bar{A}^{T} P B Q^{-1} B^{T} P \bar{A}+h Q .
$$

Finally, using the Schur Complements, one can easily observe that condition (15) of Theorem 1 guaratees $\dot{V}(y(t))<0$. 
We have $\dot{V}(x(t))<0$ if $S_{1}<0$, while $S_{1}<0$ if and only if condition (15) holds. Therefore, system (13) is exponentially stable with decay rate $\alpha$.

\section{Illustrative examples}

Example 3. Consider the linear delay system (10), where

$$
\begin{gathered}
m=2, \quad h_{1}=0.5, h_{2}=1, h=1, \\
A_{0}=\left(\begin{array}{cc}
-2 & 1 \\
-2 & -4
\end{array}\right), \quad A_{1}=\left(\begin{array}{cc}
0.1 & 0 \\
0 & 0.1
\end{array}\right), \quad A_{2}=\left(\begin{array}{cc}
0.05 & 0 \\
0 & 0.05
\end{array}\right),
\end{gathered}
$$

with $\alpha=0.8$ and $\varepsilon=0.01$. Then the matrices $A_{1}, A_{2}$ are converted to diagonal form and subtracting $\varepsilon>0$ from each diagonal entry. We choose the matrix with maximum norm which is denoted by $G_{\varepsilon}$. The remaining matrix is denoted by $B_{\varepsilon}$, where

$$
G_{\varepsilon}=\left(\begin{array}{cc}
0.09 & 0 \\
0 & 0.09
\end{array}\right), \quad B_{\varepsilon}=\left(\begin{array}{cc}
0.01 & 0 \\
0 & 0.01
\end{array}\right) \text {. }
$$

Therefore from system (10) we have

$$
\begin{gathered}
x(t)=A_{0} x(t)+\left(G_{\varepsilon}+m B_{\varepsilon}\right) x(t-h), t \in \mathbb{R}^{+}, \\
x(t)=\varphi(t), t \in[-h, 0],
\end{gathered}
$$

where the matrix functions $A_{0},\left(G_{\varepsilon}+m B_{\varepsilon}\right)$ are constant matrices. Let us set

$$
\begin{gathered}
B=\left(G_{\varepsilon}+m B_{\varepsilon}\right), \\
B_{0}=A_{0}+\alpha I, \\
\bar{A}=B_{0}+B e^{\alpha h} .
\end{gathered}
$$

Applying condition (15) Theorem 2, with $Q=I$ then we have a positive definite solution

$$
P=\left(\begin{array}{cc}
0.21 & 0.049 \\
0.049 & 0.1075
\end{array}\right)
$$

According Theorem 2, the system is 0.8 -stable. This system is 0.5 -stable by [12]. The stability of the system depends on $\varepsilon$. When the positive number $\varepsilon$ decreases we obtain an improvement of the result.

Example 4. Consider the linear delay system

$$
\begin{gathered}
x(t)=A_{0} x(t)+A_{1} x(t-1)+A_{2} x(t-\sqrt{3}), \quad t \geq 0, \\
x(t)=\phi(t), \quad t \in[-\sqrt{3}, 0],
\end{gathered}
$$

where

$$
A_{0}=\left(\begin{array}{cc}
-7 & -1 \\
0.5 & -5.5
\end{array}\right), \quad A_{1}=\left(\begin{array}{cc}
10.1 & 14.2 \\
-6.6 & -10.2
\end{array}\right), \quad A_{2}=\left(\begin{array}{cc}
-5.2 & -6.2 \\
3.6 & 5.1
\end{array}\right)
$$


with $\alpha=0.7$ and $\varepsilon=0.01$. Then the matrices $A_{1}, A_{2}$ are converted into diagonal form and subtracting $\varepsilon>0$ from each diagonal entry. We choose the matrix with maximum norm which is denoted by $G_{\varepsilon}$. The remaining matrix is denoted by $B_{\varepsilon}$, we have

$$
G_{\varepsilon}=\left(\begin{array}{cc}
-0.227 & 0 \\
0 & 0.265
\end{array}\right), \quad B_{\varepsilon}=\left(\begin{array}{cc}
0.01 & 0 \\
0 & 0.01
\end{array}\right) \text {. }
$$

Therefore from system (10) we have

$$
\begin{gathered}
\dot{x}(t)=A_{0} x(t)+\left(G_{\varepsilon}+m B_{\varepsilon}\right) x(t-h), t \in \mathbb{R}^{+}, \\
x(t)=\varphi(t), t \in[-h, 0],
\end{gathered}
$$

where the matrix functions $A_{0},\left(G_{\varepsilon}+m B_{\varepsilon}\right)$ are constant matrices. Let us set

$$
\begin{gathered}
B=\left(G_{\varepsilon}+m B_{\varepsilon}\right), \\
B_{0}=A_{0}+\alpha I, \\
\bar{A}=B_{0}+B e^{\alpha h} .
\end{gathered}
$$

Applying condition (15) Theorem 2 with $Q=I$, we have

$$
P=\left(\begin{array}{cc}
1 & 0 \\
0 & 2.421
\end{array}\right) \text {. }
$$

According to Theorem 2, the system is 0.7 -stable. This system is 0.09 -stable by [8].

\section{Conclusion}

In this brief we converted a system of multiple time delays into a single time delay system and showed that if the old system is stable, then the new one is so. Then we consider the exponential stability problem for linear systems with single delay, based on the Lyapunov-Kradovskii functional approach and (LMI). Numerical examples show the effectiveness of theoretical results.

\section{Acknowledgements}

a) Authors are very grateful to thank to one of the reviewers for carefully reading the paper and for their comments and suggestions which have improved the paper.

b) The first author was suppoted by Alzahra University.

\section{References}

1. Phat Vu N., Niamsup P., "Stability of linear time-varying delay systems and applications to control problems", J. Comput. Math. Appl. 194 (2006) 343-356. 
2. Su J. H., "Further results on the robust stability of linear systems with a single time delay", Syst. Control Lett. 23 (1994) 375-379.

3. Boyd S., Ghaoui EL., Feron E., Balakrishon V., "Linear matrix inequality and control theory", SIAM Studies in Applied Mathematics vol 15, SIAM, Philadelphia, PA (1994).

4. Kharitonov V. L., "Lyapunov-Krasovskii functional for scalar time delay equations", Syst. Control, Lett. 51 (2004) 133-149.

5. Xu S., Lam J., "Improved delay-dependent stability criteria for time-delay systems", IEEE Trans. Automat. Control 50 (2005) 384-387.

6. Xu B., "Stability criteria for linear systems with uncertain delays", J. Math. Anal. Appl. 284 (2003) 455-470.

7. Liu P. L., Su T. J., "Roboust stability of interval time-delay systems with delay-dependence", Syst. Control Lett. 33 (1998) 231-239.

8. Ren F., Cao J., "Novel $\alpha$-stability criterion of linear systems with multiple time delays", J. Comput. Math. Appl. 181 (2006) 282-290.

9. Sedaghat S., Ordokhani Y., "Stability and numerical solution of time variant linear systems with delay in both the state and control", Iran. J. Math. Sci. Inform. 7(1) (2012) 43-57.

10. Behmardi D., Ordokhani Y., Sedaghat S., "Delay-dependent robust asymptotically stable for linear time variant systems", Amirkabir J. Mod. Simul. 41(1) (2009) 35-40.

11. Yue D., Won S., "Delay-dependent robust stability of stochastic systems with time delay and nonlinear uncertaities", Electron. Lett. 37 (2001) 992-993.

12. Tan M.C., "Asymptotic stability of nonlinear systems with unbounded delays", J. Comput. Math. Appl. 337 (2008) 1010-1021.

13. wang S., Jia Z., "Inequality in matrix theory, Anhui Education Press", Hefei, 1994 (in Chines).

14. Landry M., Campbell S.A., Morris K., Aguilar C., "Dynamics of an inverted pendulum with delay feedback control", SIAM J. Applied Dynamical Systems, 4 (2005) 333-351.

15. Attia E., Bondor M., Forys U., "Angiogenesis models with discrete delays, Math. Biosci. Eng., 14(1) (2017) 1-15.

16. Bugong X., "Stability criteria for linear systems with multiple time-varying delays", J. Control Theory Appl. 1 (2003) 65-69. 